\title{
A novel flush technique to simulate natural dispersal of spermatozoa in the female reproductive tract and expedite motility assessment of fresh ejaculated Merino (Ovis aries) sperm
}

\author{
N. H. Boshoff ${ }^{1}$, H. Lambrechts ${ }^{1 \#}$, L. Maree ${ }^{2}$, S. W. P. Cloete ${ }^{1,3}$ \& G. van der Horst ${ }^{2}$ \\ ${ }^{1}$ Department of Animal Sciences, Stellenbosch University, P/Bag X1, Matieland, 7602, South Africa; \\ ${ }^{2}$ Department of Medical Biosciences, University of the Western Cape, P/Bag X17, Bellville, 7535, South Africa; \\ ${ }^{3}$ Directorate Animal Sciences: Elsenburg, P/Bag X1, Elsenburg, 7607, South Africa
}

(Received 23 June 2017; Accepted 25 January 2018; First published online 15 February 2018)

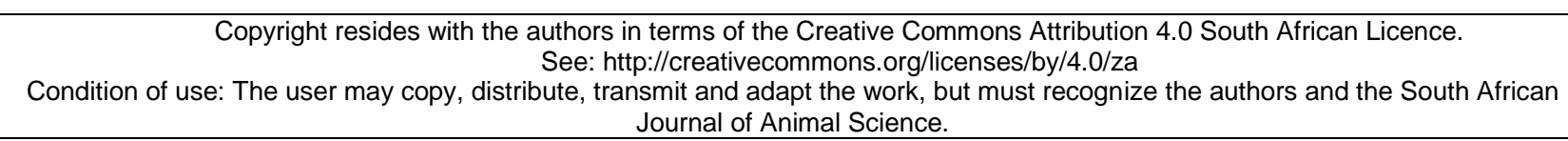
Journal of Animal Science.

\begin{abstract}
Motility is an important criterion in the assessment and quantification of the quality of ejaculated and epididymal sperm samples. Ovine sperm spermatozoa are particularly susceptible to cryodamage, and shortening the interval from collection to cryostorage may potentially minimize the negative effects of cryopreservation, thereby improving the post-thaw viability of ram spermatozoa. The use of the swim-up technique (SUT) in quantifying spermatozoa motility is well documented, especially for the isolation of highly motile spermatozoa for assisted reproductive purposes. However, this technique is time consuming and involves a swim-up period of 10 minutes before the motility of a sample is recorded by using computerassisted sperm analysis (CASA) software such as the Sperm Class Analyser $\left.\AA^{(S C A} \AA\right)$. The novel flush technique (FT) allows for capturing of sperm motility tracks via the SCA® system shortly after semen collection, that is, within a minute. This study compared fresh ejaculated sperm motility traits by using the SUT and FT. Motility evaluations were performed using 45 semen samples obtained from 15 adult Merino rams (Ovis aries) at weekly intervals. Motility recordings were captured at 100 frames per second, using a calibrated $20 \mu \mathrm{m}$ deep Leja slide. The percentage total motile spermatozoa of samples subjected to the FT was $89.2 \%$, which was significantly higher than that recorded by the SUT (83.9\%). The results also indicated that the wobble (WOB) parameter showed significantly higher values when using the FT, and parameters curvilinear velocity (VCL) and amplitude of the lateral head displacement (ALH) indicated significantly higher values when using the SUT. Establishing the ideal spermatozoa concentration and analysis of sperm subpopulation motility characteristics would assist in the optimization of the FT, and its use in CASA motility analysis of ovine spermatozoa. Standardization of CASA analysis of ovine semen samples, which would enable the selection of quality spermatozoa samples for use in field insemination (fresh samples) or in vitro fertilization programs, and laparoscopic Al cryopreserved samples warrants further investigation.
\end{abstract}

Keywords: Cryopreservation, merino, sperm class analyser, sperm motility, sperm separation technique

\#Corresponding author: helet@sun.ac.za

\section{Introduction}

Modern livestock production systems are increasingly incorporating assisted reproductive techniques (ARTs) as part of management programs to optimize reproductive efficiency and increase the number of progeny from high genetic merit animals. These techniques allow for the dissemination of germplasm, nationally and internationally, ensuring genetic integrity of livestock populations (Madan, 2005; Alexander et al., 2010; Van Arendonk, 2011; López-Saucedo et al., 2012; Hafez, 2015). The successful application of artificial insemination (AI), considered the most common ART, relies on the use of good quality sperm, with progressive motility, normal morphology and acrosome integrity being the most important criteria to consider when processing sperm samples for this purpose. Sperm motility is linked to viability, which ultimately 
determines the ability of sperm to migrate through the female reproductive tract to locate the oocyte and successfully fertilize it (Herman et al., 1994; Suarez \& Pacey, 2006).

Objective measurement and quantification of sperm motility parameters using CASA instruments is preferred to subjective manual sperm motility assessment (Rodriquez-Martinez et al., 2008; Tsakmakidis et al., 2010; Amann \& Waberski, 2014). Computer-aided sperm analysis systems digitize the lateral movement and trajectories of sperm, which provide more detailed information about the motility status of sperm (Rodriquez-Martinez et al., 2008).

Ram spermatozoa are particularly susceptible to cryodamage (Salamon \& Maxwell, 2000; Gundogan et al., 2010; Leahy \& Gadella, 2011). Ram spermatozoa processing protocols are not as refined, when compared with bovine protocols, to minimize the degree of cryodamage. There is thus a need to improve sheep spermatozoa processing protocols to increase the number of viable sperm post thaw. Decreasing the time from collection to storage, whether short or long term, may allow for the preservation of spermatozoa integrity before and after processing. One aspect of pre-storage processing that could be shortened involves the assessment of pre-equilibration motility.

There are various techniques to assess pre-storage motility, but there is still controversy whether such methods compromise sperm viability and fertilizing ability, eventually resulting in lower sperm recovery rates (Ricci et al., 2009). The use of the SUT, a separation technique for isolating populations of highly motile spermatozoa for use in ARTs such as in vitro fertilization (IVF), is well documented (Arny \& Quagliarello, 1987; Parrish \& Foote, 1987; Ing et al., 1991; Shamsuddin \& Rodriquez-Martinez, 1994; García-López et al., 1996; Jameel, 2008; Mehmood et al., 2009). However, a limitation of the SUT is the long interval from semen collection to the recording of sperm motility, which may impair post-thaw sperm viability and fertilizing ability. The flush technique $\left(\mathrm{FT}^{1}\right)$, which analyses sperm motility of vertebrate and invertebrate species almost immediately after semen collection (Van der Horst et al., 2010; Van der Horst et al., 2012) may possibly allow for a more rapid assessment of the motility of ram spermatozoa, thereby minimising deleterious changes that can occur during sperm processing. The way in which spermatozoa are processed for evaluation is equally important, because the standard dilution of spermatozoa with a medium may present a potential source of physiological stress to the spermatozoa. Techniques such as the SUT and FT, in which spermatozoa are gradually exposed to the medium, and in part the gradual dispersal of sperm in the vagina and cervix is simulated, may present a more realistic approach to studying spermatozoa motility with CASA.

The aim of this study was therefore to assess the potential of the FT to quantify pre-equilibration motility of fresh ejaculated spermatozoa, and to compare the results with those obtained from a standard pre-equilibration sperm separation technique. The study hypothesis is that the FT will allow for a more rapid assessment of the motility of ram spermatozoa before equilibration and liquid or cryopreserved storage, thereby optimising the viability of the sample for use in field insemination, in vitro embryo production programs or laparoscopic Al.

\section{Materials and methods}

Ethical clearance was obtained from the Departmental Ethics Committee for Research on Animals (DECRA) of the Department of Agriculture, Western Cape, South Africa (DECRA reference number R11/45). The experiments were conducted during autumn (southern hemisphere) on Elsenburg Research Farm $\left(33^{\circ} 51^{\prime} \mathrm{S}, 18^{\circ} 50^{\prime} \mathrm{E}\right)$, outside Stellenbosch in the Western Cape, South Africa.

To simulate industry conditions, sexually mature Merino rams $(n=15)$ aged between two and five years were used in this study. The rams were maintained in a single flock according to ethically approved husbandry practices and under uniform nutritional conditions, that is grazing on irrigated kikuyu (Pennisetum clandestinum) pastures. All animal care and procedures used in the study were in accordance with the South African National Standard 10386:2008.

All rams, regardless of age, were trained to ejaculate into an artificial vagina according to the method described by Brady \& Gildow (1939), Hafez \& Hafez (2008) and Boshoff (2014). Water temperature in the lining ranged between $42^{\circ} \mathrm{C}$ and $45^{\circ} \mathrm{C}$. A total of 45 semen samples were collected from trained rams, over three weeks. Semen samples were collected from a maximum of five rams per collection session to allow for analyses to be carried out during each session. Immediately after collection, semen samples were prepared for motility assessment using the SCA® CASA system version 5.1 (Microptic S.L., Barcelona, Spain). Each sample was subjected to the FT and SUT treatments, and prepared as described below for motility analysis using the SCA® system. The SUT and FT analyses were both performed on a heated microscope stage (37 $\left.{ }^{\circ} \mathrm{C}\right)$.

For the SUT, $0.5 \mathrm{~mL}$ pre-warmed Ham's F10 $\left(37^{\circ} \mathrm{C}\right)$ (Sigma-Aldrich, South Africa) was transferred to an Eppendorf tube. An aliquot $(10 \mu \mathrm{L})$ of each fresh semen sample was then carefully deposited at the

\footnotetext{
${ }^{1}$ Developed by Gerard van der Horst (gvdhorst@uwc.ac.za).
} 
bottom of the Eppendorf tube, underneath the Ham's F10. The semen-Ham's F10 preparation was then incubated at $37^{\circ} \mathrm{C}$ for 10 minutes, which allowed for the most motile and viable spermatozoa to swim upwards into the Ham's F10 layer, forming a cloudy area at the top of the preparation. After incubation, an aliquot $(4.5 \mu \mathrm{L})$ of motile spermatozoa was collected from the cloudy layer, and loaded into a pre-warmed 20 $\mu \mathrm{m}$ deep Leja® slide (Leja Products B.V., Nieuw-Vennep, The Netherlands).

For the FT, an aliquot $(0.2 \mu \mathrm{L})$ of fresh semen was introduced at the opening of a $20 \mu \mathrm{m}$ deep Leja ${ }^{\circledR}$ slide chamber. The fresh semen was then 'flushed' into the chamber at the point of entry using $4 \mu \mathrm{L}$ of prewarmed Ham's F10 solution $\left(37^{\circ} \mathrm{C}\right)$, which resulted in an uneven distribution of the sample through the chamber. The chamber content was allowed to stabilize on the heated microscope stage for 20 seconds before sperm motility was recorded at 100 frames per second. During a test run, it was observed that the FT creates a gradient in terms of spermatozoa dispersal, where the distal end of the chamber contained mainly semen with little mixing of Ham's F10 medium. Raw ram semen samples are characterized by the presence of fine seminal plasma granules. Care was thus taken to record motility in areas where such granules were absent (clear optical background) and where spermatozoa were evenly distributed (closer to the middle of the chamber), with the latter serving as a landmark that spermatozoa were now predominantly exposed to the medium. The time that elapsed between loading the Leja $®$ chambers and recording motility was less than a minute per sample.

Sperm motility was recorded using the motility and concentration module of SCA® software, version 5.1 (Microptic S.L., Barcelona, Spain). Data capturing involved the use of a black and white fast rate Basler A602f fire-wire digital camera (Microptic S.L., Barcelona, Spain), which was mounted (C-mount) on an Olympus $\mathrm{CH} 2$ microscope (Wirsam, Cape Town, South Africa), equipped with negative phase contrast optics $(10 \times$ objective $)$ and a heated stage $\left(37^{\circ} \mathrm{C}\right)$. Motility and kinematic parameters assessed in this study are presented in Table 1, and the software settings are summarized in Table 2. It was difficult in both the SUT and the FT to capture fields with equal spermatozoa concentrations, since semen was not diluted as in standard CASA procedures. It was accordingly necessary to record fields for each of the techniques, which ranged in sperm concentration from 20 to $40 \mathrm{million} / \mathrm{mL}$. Alternatively, each recorded field contained about 150 to 300 spermatozoa, and usually more than 500 spermatozoa were captured for each data point.

Table 1 Description of spermatozoa motility parameters assessed in this study (Boshoff, 2014)

\section{Motility parameter}

\section{Description}

Total motility (\%)

Progressive motility (\%)

Curvilinear velocity $(\mathrm{VCL})(\mu \mathrm{m} / \mathrm{s})$

Average path velocity (VAP)

$(\mu \mathrm{m} / \mathrm{s})$

Straight-line velocity (VSL)

$(\mu \mathrm{m} / \mathrm{s})$

Amplitude of the lateral head displacement $(\mathrm{ALH})(\mu \mathrm{m})$

Straightness of average special path (STR) (\%)

Linearity of curvilinear trajectory

(LIN) $(\%)$

Oscillation index value (WOB)

(\%)

Beat-cross frequency $(\mathrm{BCF})(\mathrm{Hz})$
Percentage of spermatozoa on field showing motility, classified into fast, medium, or slow motility, and thus also the number of static/immotile spermatozoa Percentage of spermatozoa moving progressively forward

Time-average velocity of a sperm head along its actual curvilinear trajectory Time-average of a sperm head along the spatial average trajectory of spermatozoa

Time-average velocity of a sperm head along the straight line between the first detected position and the last detected position

Magnitude of the lateral displacement of a sperm head about its spatial average trajectory. It can be expressed as a maximum or an average of these displacements

Measurement of the linearity of the spatial average path, calculated as VSL divided by VAP

Measurement of the linearity of the curvilinear trajectory, calculated as VSL divided by VCL

Measurement of the oscillation of the actual trajectory, calculated as VAP divided by VCL

Time-average rate at which the curvilinear sperm trajectory crosses its average path trajectory

Statistical analysis was performed using PROC GLM of SAS Enterprise Guide 5.1 software, version 9.3 of the SAS System for Windows. The generalized linear model (GLM) procedure was used to estimate least square means (LSM). These means were adjusted for multiple comparisons with the Bonferroni t-test. For comparative analysis of the FT and SUT, the one-way analysis of variance (ANOVA) approach was used, with repeated sampling per animal incorporated into the statistical model. Results were expressed as the mean \pm standard error of the mean (SEM). The variability of each motility parameter was assessed by 
providing coefficients of variation. Differences in mean parameter values recorded for the two techniques were considered statistically significant at $P<0.05$.

Table 2 Capturing and analytical software settings for the SCA® system used in the study. Cut-off points for VCL categories as recommended by Maree \& Van der Horst (2013)

\section{CAPTURING PROPERTIES}

Number of images
Images/second
Optics
Chamber
Automatic analysis

ANALYTICAL PROPERTIES

Particle area $\left(\mu \mathrm{m}^{2}\right)$
VCL Slow $(\mu \mathrm{m} / \mathrm{s})$
VCL Medium $(\mu \mathrm{m} / \mathrm{s})$
VCL Rapid $(\mu \mathrm{m} / \mathrm{s})$
Progressive $(\% S T R)$
Circular $(\% L I N)$
Connectivity
VAP Points @100 images/second

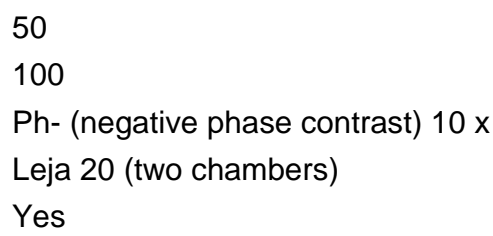

\section{Minimum}

3

$50 \quad 80$

$80 \quad 180$

180

80

$\begin{array}{ll}- & 50\end{array}$

14

11

Maximum
100
80
180
-
-
50

VCL: Curvilinear velocity; STR: Straightness of average special path; LIN: Linearity of curvilinear trajectory; VAP: Average path velocity

\section{Results and Discussion}

Motility and kinematic parameters recorded for the samples processed by the SUT and FT are presented in Table 3 . These results present the first known report of ovine sperm motility data captured at 100 frames per second.

The percentage total motile spermatozoa recorded for the FT was higher than that recorded for the SUT $(89.23 \pm 1.32 \%$ vs. $83.97 \pm 1.32 \% ; P=0.006)$. The lower total motility for the SUT could be ascribed to the time that elapsed from semen collection to recording of motility. The time that spermatozoa spend in the swim-up medium may lead to oxidative stress and thus the production of reactive oxygen species (ROS), which may affect motility detrimentally (Vishwanath \& Shannon, 1997). The production of ROS can negatively affect the resilience of spermatozoa to normal metabolic changes that influence plasma membrane and DNA integrity (Aurich, 2005). The dilution of the sample during the SUT could also result in a dilution of protective proteins in the seminal plasma, which would inhibit the spermatozoa's ability to be resilient to the changes induced by ROS that are produced during normal sperm metabolism (Soleilhavoup et al., 2014). The use of the SUT, although it favours the selection of the most motile spermatozoa in a sample, may result in spermatozoa being discriminated against in the female reproductive tract. Highly motile spermatozoa are potentially closer to the point of undergoing capacitation, and this results in destabilization of the sperm plasma membrane. Spermatozoa selected by means of the SUT can also behave differently in the cervix and oviduct secretions of the female reproductive tract, which may cause an impaired ability to travel to the site of fertilization. The female reproductive tract has developed several mechanisms to select only the most viable spermatozoa to participate in fertilization (Holt \& Fazeli, 2016).

The percentages of total motile spermatozoa recorded in this study for both the SUT $(89.23 \pm 1.32 \%)$ and FT $(83.97 \pm 1.32 \%)$ were considerably higher than those reported by Munyai $(2012)$ which ranged between $37.1 \pm 19.9 \%$ and $74.9 \pm 11.0 \%$, and Palacín et al. (2013), namely, $53.6 \pm 1.7 \%$, using an ISAS slide. In that study, motility was recorded in cases in which an increased sperm number was associated with a reduction in progressive forward movement of spermatozoa. In the current study, a raw (undiluted) sperm sample was loaded into the chamber, and accordingly sperm concentration in the FT recordings was higher than that for the SUT. 
Table 3 Comparison of motility and kinematic parameters (mean \pm SEM) recorded for fresh ejaculated ram spermatozoa prepared for SCA analysis by using the flush and the swim-up techniques, captured at 100 frames per second

\begin{tabular}{lcccc}
\hline \multirow{2}{*}{ Parameter } & \multicolumn{2}{c}{ FT (n=45) } & SUT (n=45) \\
\cline { 2 - 5 } & \multicolumn{1}{c}{ Range (CV) } & Mean \pm SEM & Range (CV) & Mean \pm SEM \\
\hline Total motility (\%) & $50.10-98.78(10.07)$ & $89.23^{\mathrm{c}} \pm 1.32$ & $48.25-97.24(10.66)$ & $83.97^{\mathrm{d}} \pm 1.32$ \\
Progressive motility (\%) & $9.39-71.78(34.23)$ & $44.8^{2} \pm 2.26$ & $5.23-79.83(32.29)$ & $47.34 \pm 2.25$ \\
Non-progressive motility $\%)$ & $21.98-78.18(35.11)$ & $44.41^{\mathrm{a}} \pm 2.30$ & $11.34-81.89(42.41)$ & $36.63^{\mathrm{b}} \pm 2.29$ \\
Rapid $(\%)$ & $7.44-86.46(21.15)$ & $61.66 \pm 1.92$ & $15.86-82.38(20.86)$ & $62.28 \pm 1.92$ \\
Medium $(\%)$ & $6.50-57.26(36.01)$ & $22.58^{\mathrm{c}} \pm 1.20$ & $4.15-55.84(50.95)$ & $15.90^{\mathrm{d}} \pm 1.19$ \\
Slow $(\%)$ & $0.42-17.29(54.46)$ & $4.99 \pm 0.40$ & $1.26-16.33(46.76)$ & $5.79 \pm 0.40$ \\
VCL $(\mu \mathrm{m} / \mathrm{s})$ & $123.27-329.41(16.35)$ & $244.32^{\mathrm{a}} \pm 5.89$ & $141.83-389.61(15.08)$ & $263.96^{\mathrm{b}} \pm 5.87$ \\
VSL $(\mu \mathrm{m} / \mathrm{s})$ & $44.00-168.14(20.88)$ & $111.55 \pm 3.43$ & $45.48-186.90(19.39)$ & $119.65 \pm 3.42$ \\
VAP $(\mu \mathrm{m} / \mathrm{s})$ & $67.31-201.20(14.00)$ & $148.55 \pm 3.07$ & $93.15-205.46(13.45)$ & $154.01 \pm 3.05$ \\
LIN $(\%)$ & $26.09-60.02(16.69)$ & $45.44 \pm 1.12$ & $23.74-59.00(16.62)$ & $45.45 \pm 1.11$ \\
STR $(\%)$ & $48.66-84.93(11.92)$ & $74.13 \pm 1.30$ & $47.15-90.97(11.45)$ & $76.89 \pm 1.30$ \\
WOB $(\%)$ & $48.88-70.93(7.62)$ & $60.85^{\mathrm{a}} \pm 0.68$ & $49.63-68.25(7.85)$ & $58.87^{\mathrm{b}} \pm 0.68$ \\
ALH $(\mu \mathrm{m})$ & $2.09-4.16(14.64)$ & $2.88^{\mathrm{c}} \pm 0.06$ & $2.26-4.78(12.97)$ & $3.24^{\mathrm{d}} \pm 0.06$ \\
BCF $(\mathrm{Hz})$ & $27.15-56.98(12.06)$ & $44.26 \pm 0.79$ & $18.08-59.69(11.70)$ & $45.44 \pm 0.78$ \\
& & & &
\end{tabular}

$\overline{\mathrm{a}, \mathrm{b}}$ Row means with different superscripts differ significantly at $P<0.05 ;{ }^{\mathrm{c}, \mathrm{d}}$ Row means with different superscripts differ significantly at $P<0.01$; VCL: Curvilinear velocity; VSL Straight-line velocity, VAP Average path velocity, LIN Linearity of curvilinear trajectory, STR Straightness of average special path, WOB Wobble, ALH lateral head displacement, BCF Beat-cross frequency; FT: flush technique; SUT: swim-up technique

Compared with the SUT, the FT resulted in higher values for the percentage non-progressive motile spermatozoa $(44.41 \% \pm 2.30 \%$ vs. $36.63 \% \pm 2.29 \%, P=0.019)$, and the percentage medium velocity motile spermatozoa $(22.58 \% \pm 1.20 \%$ vs. $15.90 \% \pm 1.19 \%, P=0.0002)$ (Table 3$)$. These differences were expected and could be attributed to the pre-selection of sperm subpopulations, because the SUT discriminates against slow-swimming and immotile spermatozoa (Marti et al., 2011; Maree \& Van der Horst, 2013). In contrast, the FT allows for assessment of the entire sample, including all sperm motility subpopulations, thus presenting a more accurate profile of the raw semen. When the fertilizing ability of ram semen needs to be evaluated, the analysis of the entire sperm population would provide a more accurate indication of the quality of such a sample. Studies correlating CASA-assessed motility with field fertility are scarce and further research is needed to elucidate the relationship between CASA-assessed progressive forward motility and field fertility.

Spermatozoa isolated with the SUT expressed higher mean VCL values compared with spermatozoa assessed using the FT $(263.96 \mu \mathrm{m} / \mathrm{s} \pm 5.87 \mu \mathrm{m} / \mathrm{s}$ vs. $244.32 \mu \mathrm{m} / \mathrm{s} \pm 5.89 \mu \mathrm{m} / \mathrm{s} ; P=0.0206)$. The FT showed a higher WOB value when compared with the SUT $(60.85 \% \pm 0.68 \%$ vs. $58.87 \% \pm 0.68 \%)(P=0.043)$, but a lower mean lateral head displacement $(\mathrm{ALH})$ value $(2.88 \mu \mathrm{m} \pm 0.06 \mu \mathrm{m}$ vs. $3.24 \mu \mathrm{m} \pm 0.06 \mu \mathrm{m} ; P=0.0001)$. The higher ALH and VCL values and lower WOB value reported for the SUT could be ascribed to the selection of the most motile sperm population by the SUT. In addition, the lower sperm concentration in the chamber could have resulted in more space being available for spermatozoa to swim unhindered, compared with the higher concentration with the FT, where the frequency of spermatozoa encountering one another and thus interfering with one another's swimming trajectory would be higher. Palacín et al. (2013) found that in the ISAS chamber, spermatozoa tended to move in different focal planes, which would influence the ability of the CASA system to reflect motility accurately. Verstegen et al. (2002) also highlighted this limitation of a too high chamber spermatozoa concentration. Even though spermatozoa concentration was determined only after the $\mathrm{SCA} \AA$ recordings were made in the current study, care was taken with both sperm preparation techniques to capture only fields in which sperm concentrations ranged between 20 and $40 \mathrm{million} / \mathrm{mL}$.

The use of CASA as a tool to assess the potential of ram sperm samples for use in ART programs still warrants considerable research (Holt et al., 2007; Acharya et al., 2017). The potential effect of sperm 
subpopulation structure, that is, degree of homogeneity or heterogeneity, needs to be taken into account when sperm motility is assessed. Although mostly motile spermatozoa were loaded into the chamber during the SUT, the differences between subpopulations in terms of stage of maturation and acquisition of the ability to swim may influence the ability of the spermatozoa to move around freely in the chamber (Bravo et al., 2011). In their study, five distinct subpopulations could be identified, which ranged from rapid progressive and non-sinuous spermatozoa (VCL: $126.41 \mu \mathrm{m} / \mathrm{s}$ ) to poorly motile, non-progressive spermatozoa (VCL: $36.81 \mu \mathrm{m} / \mathrm{s})$.

It is evident from this study, as was reported by Yimer et al. (2009), that the optimal dilution rate for raw sperm samples needs to be determined to ensure the effective use of CASA to analyse ram sperm motility. To ensure the success of Al and multi-ovulation embryo transfer programs in sheep, the use of good-quality high-density sperm samples with good fertilizing potential is required. For IVF protocols, in which frozen-thawed sperm are used most often, sperm selection methods are normally used to select the most motile viable spermatozoa, and inadvertently eliminate the potential inhibiting effect of seminal plasma and minimize or prevent the toxic and lithic effects caused by dead and/abnormal spermatozoa (Aria et al., 2017). This would not be the case where raw spermatozoa samples are just diluted with skim milk, and stored at $4{ }^{\circ} \mathrm{C}$ until used for field inseminations. With liquid storage, a potential disadvantage is the formation of ROS, which when produced at the improper time in a sperm's lifetime, would have a detrimental effect on sperm membrane integrity and function (Sanocka \& Kurpisz; 2004; Mata-Campuzano et al., 2012).

The inconsistency between the CASA parameter values obtained in this study with those reported in the studies of Robayo et al. (2008), Munyai (2012), and Palacín et al. (2013) emphasizes the importance of standardizing CASA assessment protocols to allow for comparison of studies from different laboratories. Standardization would enable researchers to potentially correlate CASA results to the in vivo scenario of sperm transport in the female reproductive tract. Factor, such as dilution medium, season, collection method, breed, different CASA systems, and software analysis setting, can all influence the values obtained.

\section{Conclusions}

The findings of this study indicated that the FT produced results that compared favourably with those obtained with the SUT. The FT can thus be considered an alternative approach to the pre-equilibration assessment of sperm motility. However, the influence of chamber sperm density and sperm subpopulation structure warrant further investigation to allow for the refinement of the FT. Optimizing the dilution rate of semen samples would allow for uninhibited spermatozoa movement in the Lejaß chamber, and more accurate assessment of motility. Refinement of the FT would ensure a shorter pre-storage processing interval, which would minimize the production and related effects of ROS on the integrity of ram spermatozoa, thereby improving the resilience of spermatozoa to processing and cryopreservation. It is also important to establish and standardize the relationship between CASA parameters and the fertilizing ability of ram spermatozoa, for this would support the use of CASA as part of the reproductive toolbox for the sheep industry to optimize the reproductive efficiency of production systems, thereby ensuring sustainable and cost-efficient meat production.

\section{Acknowledgements}

The authors would like to thank Mrs A. Kruger and colleagues at Elsenburg Research Farm for their assistance in collection of semen samples, and Mrs G. Jordaan for assistance with the statistical analysis.

This study was supported financially by the Meat Industry Trust (MIT), and Western Cape Agricultural Research Trust (WCART). The SCA® CASA system was provided by the Comparative Spermatology Laboratory at the University of the Western Cape.

\section{Authors' Contributions}

NHB conducted the research during her MSc Agric studies and was responsible for drafting and submitting the manuscript. $\mathrm{HL}$ as supervisor critically analysed and interpreted the results and revised the original manuscript. GvdH and LM as collaborators assisted with the capture of the SCA motility recordings, and the sperm motility analysis and commented on the original and revised manuscript. SWPC as co-supervisor provided the resource flock animals that were used, provided funding and contributed to the editorial care of the thesis by NHB, as well as the initial and revised manuscripts.

\section{Conflict of Interest Declaration}

The authors declare that there is no conflict of interest.

\section{References}

Acharya, M., Burke, J.M. \& Rorie, R.W., 2017. Effect of ram semen extenders and supplements on computer assisted sperm analysis parameters. J. Anim. Sci. 95(1). Doi: 10.2527/ssasas2017.0102. 
Alexander, B., Mastromonaco, G. \& King, W.A., 2010. Recent advances in reproductive biotechnologies in sheep and goat. J. Vet. Sci. Techn. 1, 101. Doi: 10.4172/2157-7579.1000101.

Amann, P.R. \& Waberski, D., 2014. Computer-assisted sperm analysis (CASA): Capabilities and potential developments. Theriogenology 81, 5-17.

Aria, M.E., Andara, K., Briones, E. \& Felmer, R., 2017. Bovine sperm separation by swim-up and density gradients (Percoll and BoviPure): Effect on sperm quality, function and gene expression. Reprod. Biol. 17(2), 126-132.

Arny, M. \& Quagliarello, J., 1987. Semen quality before and after processing by a swim-up method: Relationship to outcome of intrauterine insemination. Fertil. Steril. 48(4), 643-648.

Aurich, C., 2005. Factors affecting the plasma membrane function of cooled-stored stallion spermatozoa. Anim. Reprod. Sci. 89, 65-75.

Boshoff, N.H., 2014. The influence of genotype on sperm motility and sperm head morphometry of Merino (Ovis aries) sheep. MSc Agric thesis, University of Stellenbosch, South Africa.

Brady, D.E. \& Gildow, E.M., 1939. Characteristics of ram semen as influenced by the method of collection. J. Anim. Sci. $1939,250-254$.

Bravo, J.A., Montanero, J., Calero, R. \& Roy, T.J., 2011. Identification of sperm subpopulations with defined motility characteristics in ejaculates from lle de France rams. Anim. Reprod. Sci. 129, 22-29.

García-López, N., Ollero, M., Muino-Blanco, T. \& Cebrián-Pérez, J., 1996. A dextran swim-up procedure for separation of highly motile and viable ram spermatozoa from seminal plasma. Theriogenology 46(1), 141-151.

Gundogan, M., Yeni, D., Avdatek, F. \& Fidan, A., 2010. Influence of sperm concentration on the motility, morphology, membrane and DNA integrity along with oxidative stress parameters of ram sperm during liquid storage. Anim. Reprod. Sci. 122(3-4), 200-207.

Hafez, B. \& Hafez, E.S.E., 2008. Reproduction in farm animals.7th edition. Wiley-Blackwell, USA.

Hafez, Y.M., 2015. Assisted reproductive technologies in farm animals. ICMALPS 2015, Alexandria University, Egypt.

Herman, H.A., Mitchell, J.R. \& Doak, G.A., 1994. The artificial insemination and embryo transfer of dairy and beef cattle (including information pertaining to goats, sheep, horses, swine, and other animals). 8th edition. Interstate Publishers, Inc., Kansas, USA.

Holt, W.V. \& Fazeli, A., 2016. Sperm selection in the female mammalian reproductive tract. Focus on the oviduct: hypotheses, mechanisms, and new opportunities. Theriogenology 85, 105-112.

Holt, W.V., O'Brien, J. \& Abaigar, T., 2007. Applications and interpretation of computer-assisted sperm analyses and sperm sorting methods in assisted breeding and comparative research. Reprod. Fert. Developm. 19: 709-718.

Ing, R.M., Li, D.Q., Harding, A.M. \& Jones, W.R., 1991. A comparison of swim-down and swim-up methods for the extraction of high motility sperm. Fertil. Steril. 55(4), 817-819.

Jameel, T., 2008. Sperm swim-up: A simple and effective technique of semen processing for intrauterine insemination. J. Pakistan Med. Ass. 58(2), 71-74.

Leahy, T. \& Gadella, B.M., 2011. Sperm surface changes and physiological consequences induced by sperm handling and storage. Reproduction 142(6), 759-778.

López-Saucedo, J., Paramio-Nieto, M.T., Fierro, R. \& Pina-Aguilar, R.E., 2012. Intracytoplasmic sperm injection (ICSI) in small ruminants. Anim. Reprod. Sci. 133: 129-138.

Madan, M., 2005. Animal biotechnology: Applications and economic implications in developing countries. Rev. Sci. Tech. Off. Int. Epiz. 24(1), 127-139.

Maree, L. \& Van der Horst, G., 2013. Quantification and identification of sperm sub-populations using computer-aided sperm analysis and species-specific cut-off values for swimming speed. Biotech.Histochem. 3-4, 181-193.

Martí, J.I., Aparicio, I.M. \& García-Herreros, M., 2011. Sperm morphometric sub-populations are differentially distributed in rams with different maturity age in cryopreserved ejaculates. Theriogenology 76, 97-109.

Mata-Campuzano, M., Alvarez-Rodriguez, M., Anel, L.D., Garde, J.J. \& Martinez-Pastor, F., 2012. Effect of several anitioxidants on thawed ram spermatozoa submitted to $37^{\circ} \mathrm{C}$ up to four hours. Reprod. Domest. Anim. 47, 907914.

Mehmood, A., Anwar, M. \& Naqvi, S., 2009. Motility, acrosome integrity, membrane integrity and oocyte cleavage rate of sperm separated by swim-up or Percoll gradient method from frozen-thawed buffalo semen. Anim. Reprod. Sci. 111(2), 141-148.

Munyai, P.H., 2012. Cryopreservation of South African indigenous ram semen. MSc thesis, University of the Free State, South Africa.

Palacín, I., Vicente-Fiel, S., Santolaria, P. \& Yániz, J.L., 2013. Standardization of CASA sperm motility assessment in the ram. Small Rum. Res. 112, 128-135.

Parrish, J.J. \& Foote, R.H., 1987. Quantification of bovine sperm separation by a Swim-up method relationship to sperm motility, integrity of acrosomes, sperm migration in polyacrylamide gel and fertility. J. Androl. 8(4), 259-266.

Ricci, G., Perticarari, S., Boscolo, R., Montico, M., Guaschino, S. \& Presani, G., 2009. Semen preparation methods and sperm apoptosis: swim-up versus gradient-density centrifugation technique. Fert. and Steril. 91(2), 632-638.

Robayo, I., Montenegro, V., Valdés, C. \& Cox, J.F., 2008. CASA assessment of kinematic parameters of ram spermatozoa and their relationship to migration efficiency in ruminant cervical mucus. Reprod. Dom. Anim. 43: 393-399.

Rodríguez-Martínez, H., Saravia, F., Wallgren, M., Roca, J. \& Peña, F., 2008. Influence of seminal plasma on the kinematics of boar spermatozoa during freezing. Theriogenology 70(8), 1242-1250.

Salamon, S. \& Maxwell, W.M.C., 2000. Storage of ram semen. Anim. Reprod. Sci. 62(1), 77-112.

Sanocka, D. \& Kurpisz, M., 2004. Reactive oxygen species and sperm cells. Reprod. Biol. Endocrinol. 2, 12-19. 
Shamsuddin, M., \& Rodriguez-Martinez, H., 1994. A simple, non-traumatic swim-up method for the selection of spermatozoa for in vitro fertilization in the bovine. Anim. Reprod. Sci. 36(1), 61-75.

Soleilhavoup, C., Tsikis, G., Labas, V., Harichaux, G., Kohnke, P.L., Dacheux, D.L., Guérin, Y., Gatti, J.L., de Graaf, S.P. \& Druart, X., 2014. Ram seminal plasma proteome and its impact on liquid preservation of spermatozoa. J. Proteomics 109, 245-260.

Suarez, S. \& Pacey, A., 2006. Sperm transport in the female reproductive tract. Hum. Reprod. Update 12(1), 23-37.

Tsakmakidis, I.A., Lymberopoulos, A.G. \& Khalifa, T.A.A., 2010. Relationship between sperm quality traits and fieldfertility of porcine semen. J. Vet. Sci. 11(2), 151-154.

Van Arendonk, J.A.M., 2011. The role of reproductive technologies in breeding schemes for livestock populations in developing countries. Livest. Sci. 136: 29-37.

Van der Horst, G., Bennet, M. \& Van der Horst, M., 2012. Computer aided sperm analysis in broadcast spawners: baseline studies in assessing sperm quality quantitatively. In: Proceedings: Aqua 2012, Global Aquaculture Securing our Future. Prague, Czech Republic, 1-5 September 2012. Abstract 435.

Van der Horst, G., Luther, I., Goeritz, F., Hermes, R., Dierich, J., Lueders, I., et al., 2010. Computer aided semen analysis of Loxodonta africana sperm. International elephant conservation and research symposium. Pretoria, 25-29 January 2010.

Verstegen, J., Iguer-Ouada, M. \& Onelin, K., 2002. Computer assisted semen analyzers in andrology research and veterinary practice. Theriogenology $57,149-179$.

Vishwanath, R. \& Shannon, P., 1997. Do sperm cells age? A review of the physiological changes in sperm during storage at ambient temperature. Reprod. Fert. Dev. 9, 321-331.

Yimer, N., Rosnina, A., Wahid, A., Saharee, A.A., Yap, K.C., Ganesamurthi, P. \& Fahmi, M., 2009. Sperm concentration determination: 2X-cel slide CASA vs. haemocytometer. Proc. Internat. Conf. Anim. Health and Human Safety, 6-8 December 2009. 